\title{
Avaliação da filtração da luz em câmaras escuras portáteis e sua influência no velamento dos filmes radiográficos
}

Evaluation of light filtration in portable dark chambers and its influence on the visibility of radiographic films

Evaluación de la filtración de luz en cuartos oscuros portátiles y su influencia en el velo de películas radiográficas

\section{Resumo}

Introdução: a norma RDC $\mathrm{n}^{\circ} 330$ estabelece o uso de câmara escura portátil para o processamento de radiografias intrabucais, desde que seja confeccionada em material opaco e que tenha cronômetro e termômetro para a realização de processamento radiográfico pelo método temperatura x tempo. O objetivo desse estudo foi avaliar a filtração da luz em câmaras portáteis com visor e sem visor e sua influência no velamento dos filmes radiográficos periapicais. Metodologia: Este estudo experimental testou quatro câmaras escuras portáteis das marcas, NMartinse Essence Dental VH com visor, Manoel PG toda de acrílico vermelho e uma sem visor da Delta Del Grandi. Para controle foi utilizado um quarto totalmente escuro. Os filmes periapicais utilizados foram do grupo E (Carestream e AGFA) e processados com soluções reveladora e fixadora por três minutos no revelador, 20 segundos no banho intermediário (água), seis minutos no fixador e 10 minutos no banho final. Após secas, as radiografias foram digitalizadas. Foi realizada a medida do pixel correspondente a densidade óptica da área mais escura, obtendo-se um valor entre 0 (preto) a 255 (branco) em um computador com 8bits. Resultado: Nas câmaras escuras ManoelPG e NMartins, a imagem da moeda foi visível, demonstrando velamento por luz em ambos os filmes. Na câmara escura portátil EssenceVH o velamento foi total, não sendo possível visualizar o contraste da imagem da moeda, nem mensurar a 
densidade. Conclusão: A câmara escura sem visor mostrou-se adequada para o processamento radiográfico, entretanto, as com visor ou totalmente acrílica não filtraram adequadamente a luz causando velamento.

Palavras-chave: Radiografia dentária; Radiografia digital; Filme para Raios X.

\begin{abstract}
Introduction: RDC No. 330 is the process for using a portable camera for processing intraoral radiographs, provided that it is made of opaque material and that it has a stopwatch and thermometer to perform radiographic processing using the time $\mathrm{x}$ temperature method. The aim of this study was to evaluate the filtration of light in portable cameras with viewfinder and without viewfinder and its influence on the veiling of periapical radiographic films. Methodology: This experimental study tested four portable darkrooms from brands NMartins.e Essence Dental VH with visor, Manoel PG all red acrylic and one without a visor by Delta Del Grandi. For control, a completely dark room was used. The periapical films used were from group E (Carestream and AGFA) and processed with developer and fixer solutions for three minutes in the developer, 20 seconds in the intermediate bath (water), six minutes in the fixer and 10 minutes in the final bath. After drying, such radiographs were digitized. The measurement of the pixel corresponding to the optical density of the darkest area was performed, obtaining a value between 0 (black) and 255 (white) in an 8-bit computer. Result: In the darkrooms ManoelPG and NMartins, the image of the coin was visible, demonstrating light veiling in both films. In the EssenceVH portable darkroom, there was total blurring, and it was not possible to visualize the contrast of the coin image, nor to measure the density. Conclusion: The darkroom without a viewfinder proved to be suitable for radiographic processing, however, as with viewfinder or fully acrylic, they did not filter out, causing the light to be blurred.
\end{abstract}

Keywords: Radiography, dental; Digital radiography; X-Ray film.

\title{
Resumen
}

Introducción: RDC No. 330 es el proceso de utilización de una cámara portátil para el procesamiento de radiografías intraorales, siempre que sea de material opaco y cuente con cronómetro y termómetro para realizar el procesamiento radiográfico mediante el método de temperatura x tiempo. El objetivo de este estudio fue evaluar la filtración de luz en cámaras portátiles con visor y sin visor y su influencia en el velo de películas radiográficas periapicales. Metodología: Este estudio experimental probó cuatro cuartos oscuros portátiles de las marcas NMartins.e Essence Dental VH con visor, Manoel PG todo acrílico rojo y uno sin visor de Delta Del Grandi. Para el control, se utilizó una habitación completamente oscura. Las películas periapicales utilizadas fueron del grupo E (Carestream y AGFA) y procesadas con soluciones de revelador y fijador durante tres minutos en el revelador, 20 segundos en el baño intermedio (agua), seis minutos en el fijador y 10 minutos en el baño final. Después del secado, estas radiografías se digitalizaron. Se realizó la medición del píxel correspondiente a la densidad óptica de la zona más oscura, obteniendo un valor entre 0 (negro) y 255 (blanco) en un ordenador de 8 bits. Resultado: En los cuartos oscuros ManoelPG y NMartins, la imagen de la moneda era visible, demostrando un velo de luz en ambas películas. En el cuarto oscuro portátil EssenceVH, hubo un desenfoque total, y no fue posible visualizar el contraste de la imagen de la moneda, ni medir la densidad. Conclusión: El cuarto oscuro sin visor demostró ser adecuado para el procesamiento radiográfico, sin embargo, al igual que con el visor o completamente acrílico, no se filtraron, lo que provocó que la luz se volviera borrosa.

Palabras clave: Radiografía dental; Radiografia digital; Película para Rayos X.

\section{Introdução}

A radiografia é um exame complementar muito utilizado no diagnóstico e planejamento em odontologia (Moreira, Arruda, \& Larentis, 2017; Lima et al, 2021) contudo as imagens são observadas após exposição aos raios X, assim como um processamento químico adequado (Januário, Carvalho, \& Larentis, 2020), sendo as câmaras escuras portáteis essenciais na rotina clínica do cirurgião dentista. Uma radiografia de qualidade requer conhecimento e controle do processamento (Gasparini et al., 2005), visto que densidade, contraste, velamento e nitidez influenciam diretamente na imagem (Casanova 2002; Watanabe \& Arita, 2013). O velamento é uma densidade extra, indesejável, sobreposta à densidade básica de um filme, podendo ocorrer quando o tempo de exposição é exagerado, utilização de filmes deteriorados, inadequado armazenamento, data de validade ultrapassada e pelo processamento, com o uso de soluções também deterioradas, iluminação intensa, luz de segurança inadequada e filtros impróprios (Lemke et al., 2006).

Várias técnicas radiográficas intrabucais como periapical e interproximal são propostas para o diagnóstico de alterações ósseas, cáries e periapicopatias. Com a associação dessas técnicas obtêm-se valores de sensibilidade e especificidade significativos no diagnóstico dessas lesões (Januário, Carvalho, \& Larentis, 2020). 
Falhas no processamento radiográfico podem prejudicar o diagnóstico (Moreira, Arruda, \& Larentis, 2017), além de submeter o paciente a repetições, promovendo exposição excessiva e desnecessária aos raios X, aumento do tempo clínico e gastos dispensáveis (Silva et al., 2016; da Silva et al., 2017; Freire et al., 2021). A norma RDC nº 330, de 20 de dezembro de 2019 (Brasil, 2019) estabelece o uso de câmara escura portátil para o processamento de radiografias intrabucais, desde que seja confeccionada em material opaco e que tenha cronômetro e termômetro para a realização de processamento radiográfico pelo método temperatura $\mathrm{x}$ tempo.

Entretanto, as câmaras escuras com visor de acrílico vermelho ainda são as mais utilizadas em consultórios odontológicos, para permitir o processamento através de inspeção visual (Takeshita et al., 2011).

Os filmes mais utilizados são os do tipo E de alta sensibilidade e que não deveriam receber luz que passa, através do visor ou pelo acrílico das câmaras escuras, pois apresentariam diversos graus de velamento (Tamburús, Lavrador, \& Oliveira, 1999). Estudos sobre a eficiência do polímero acrílico para filtrar adequadamente os componentes do espectro da luz, particularmente a fluorescente, nas condições usualmente utilizadas em consultórios odontológicos e, em especial, com filmes de alta sensibilidade são necessários. Dentro desse contexto, o objetivo desse estudo foi avaliar a filtração da luz em câmaras portáteis com visor e sem visor e sua influência no velamento de filmes radiográficos periapicais.

\section{Metodologia}

Este estudo experimental foi realizado nas dependências da clínica de radiologia da UNICEPLAC-DF, mediante autorização da coordenação do curso de odontologia e sob a orientação do professor da disciplina de radiologia, durante o mês de agosto de 2017.

Quatro câmaras escuras portáteis foram selecionadas, das marcas NMartinse Essence Dental VH com visor, Manoel PG toda de acrílico vermelho e uma sem visor da Delta Del Grandi. Para controle foi utilizado um quarto totalmente escuro (Figura 1). Os filmes periapicais utilizados foram do grupo $\mathrm{E}$ das seguintes marcas Carestreame AGFA.

Dez filmes periapicais sem o invólucro foram posicionados nas câmaras escuras portáteis, sobre os quais foi colocado uma moeda. Logo após, as câmaras fechadas, foram expostas a uma lâmpada fluorescente branca (Phillips T8 4000K) de 40W posicionada no teto a uma distância de $190 \mathrm{~cm}$ por três minutos (Figura 2).

Todos os filmes foram processados pelo método temperatura tempo, pelo mesmo operador, com soluções reveladora e fixadora da marca Carestream, apenas em um dia, no período vespertino. Como a temperatura aferida do revelador foi de $25^{\circ} \mathrm{C}$, os filmes permaneceram neste por 3 minutos, por 20 segundos no banho intermediário (água), seis minutos no fixador e 10 minutos no banho final (água). Após secas, as radiografias foram digitalizadas no scanner HP® Scanjet G4050, em TIF, 300 dpi e foram importadas para e software Digora ${ }^{\circledR}$ for Windoms 2.1 (Orion Corporation Soredex, Finland). Neste software foi realizada a medida do pixel correspondente a densidade óptica da área mais escura, obtendo-se um valor entre 0 (preto) a 255 (branco), em um computador com 8bits. Foi utilizado monitor de tela plana com as seguintes especificações (Monitor LCD colorido de 21,2", de 3MP, RX320 EIZO ${ }^{\circledR}$ ).

Os dados foram submetidos aos testes estatísticos de Mann-Whitney e Kruskal Wallis no software SPSS 13 com nível de significância de 5\% (p<0,05). 
Figura 1. Câmaras escuras portáteis. A. Delta Del Grandi (sem visor) B. Manoel PG· (toda de acrílico), C. NMartins·e D. Essence Dental VH· (com visores).

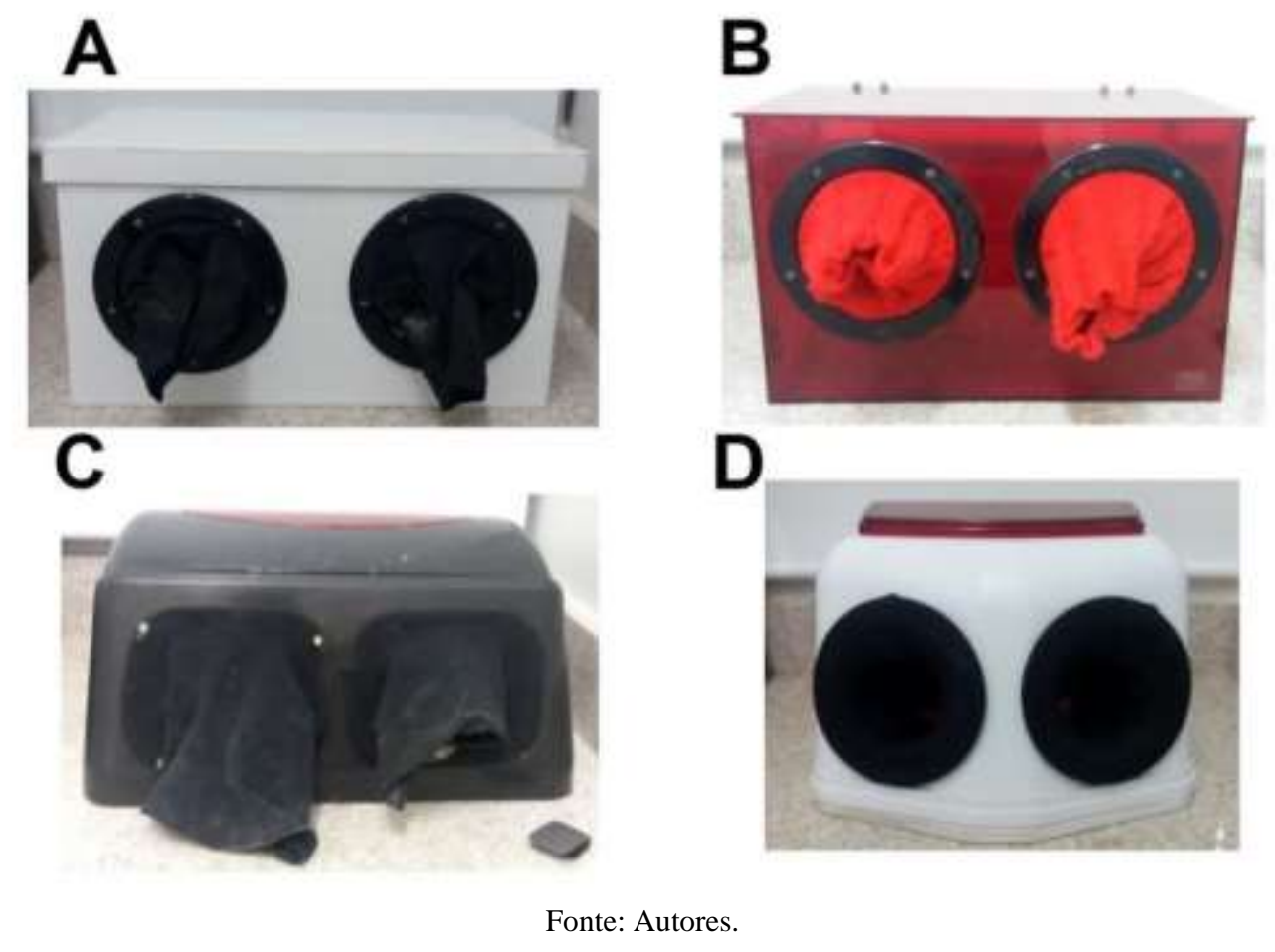

Figura 2. Filme Carestream e AGFA sem o invólucro, sob as moedas no assoalho de uma das câmaras portáteis.

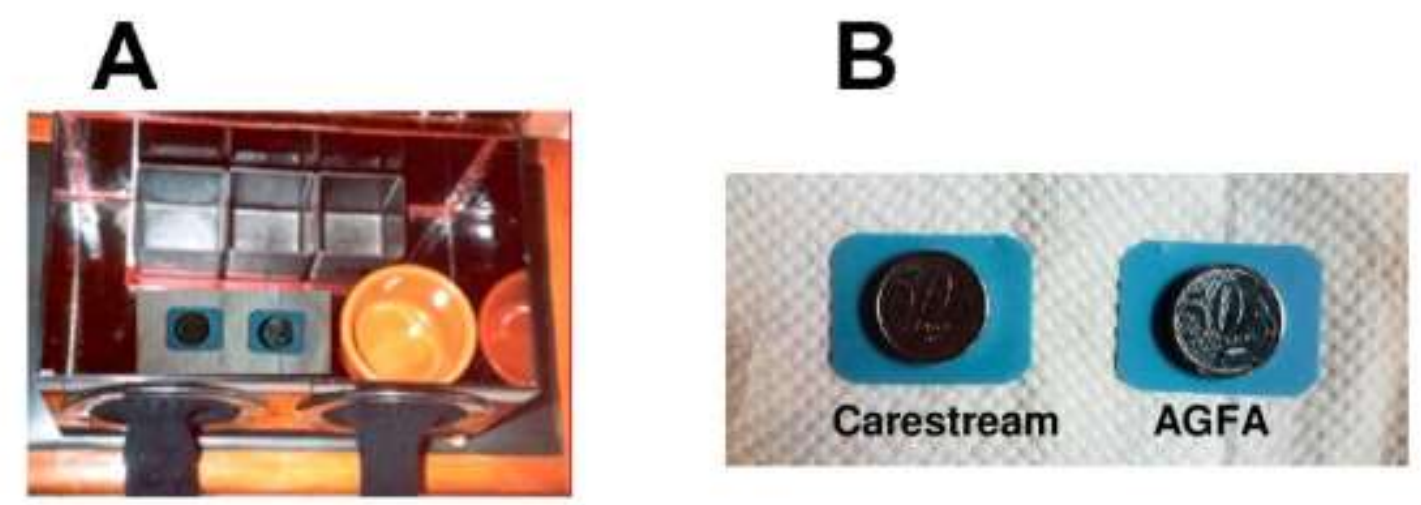

Fonte: Autores.

\section{Resultados}

Na figura 3 são visualizadas as radiografias em dois tipos de filmes, com quatro câmaras escuras e o controle. No quarto totalmente escuro, não foi observada nenhuma imagem compatível com velamento por luz, apenas a densidade base velamento (DBV). Na câmara escura Delta sem visor, também não foi visualizado velamento, entretanto o filme da AGFA apresentou maior densidade. Nas câmaras escuras ManoelPG e NMartins, a imagem da moeda foi visível, demonstrando velamento por luz em ambos os filmes. Na câmara escura portátil EssenceVH o velamento foi total, não sendo possível visualizar o contraste da imagem da moeda, nem mensurar a densidade. 
A Tabela 1 demonstra os tipos de filmes utilizados de sensibilidade E, quatro câmaras escuras portáteis e o controle. Os filmes Carestream processados no quarto totalmente escuro (controle) e na câmara Delta apresentaram resultados de densidade muito próximos, enquanto o AGFA ficou discretamente mais velado na câmara Delta. Os dois filmes apresentaram velamento muito maior nas câmaras com visor em acrílico sendo que, o Carestream se comportou um pouco melhor que o AGFA nas câmaras ManoelPG e NMartins. O velamento dos filmes da câmara escura portátil EssenceVH foi grande a ponto de não ser possível mensurar a densidade do filme.

Para apresentação dos dados, foi obtido um gráfico de densidade dos filmes processados nas câmaras escuras portáteis ManoelPG e NMartins demostrando que os filmes da AGFA apresentaram maior velamento que os da Carestream (Gráfico 1).

Figura 3. Filmes processados no quarto escuro e nas câmaras escuras portáteis: Delta Del Grandi, Manoel PG, NMartins e Essence Dental VH

\begin{tabular}{|c|c|}
\hline \multirow{2}{*}{ CÃMARAS } & \multicolumn{2}{|c|}{ FILMES } \\
\hline $\begin{array}{c}\text { CONTROLE } \\
\text { (Quarto escuro) }\end{array}$ & CARESTREAM \\
\hline $\begin{array}{c}\text { DELTA } \\
\text { (sem visor) }\end{array}$ \\
\hline $\begin{array}{c}\text { MANOEL PG } \\
\text { (com visor) }\end{array}$ \\
\hline $\begin{array}{c}\text { NMARTINS } \\
\text { (com visor) }\end{array}$ \\
\hline
\end{tabular}

Fonte: Autores. 
Tabela 1. Média +/-desvio padrão e número de observações (n) da densidade dos filmes nas diferentes câmaras escuras e no controle.

\begin{tabular}{lccc}
\hline Câmara & \multicolumn{3}{l}{ Filme } \\
\hline \multicolumn{1}{c}{ Carestream } & AGFA & p-valor* \\
Controle & $222.25 \pm 6.39(4)$ & $214.25 \pm 5.85(4)$ & 0,058 \\
Delta & $218.75 \pm 4.85(4)$ & $145.50 \pm 19.20(4)$ & 0,020 \\
Manoel PG & $177.50 \pm 40.37(4)$ & $20.25 \pm 62.27(4)$ & 0,020 \\
NMartins & $35.25 \pm 25.88(4)$ & $7.50 \pm 2.51(4)$ & 0,020 \\
Essence VH & $0.00 \pm 0.00(4)$ & $0.00 \pm 0.00(4)$ & $* * *$ \\
p-valor** & 0,002 & 0,002 & \\
\hline
\end{tabular}

(*) Teste de Mann-Whitney (**) Kruskal Wallis (***)

Fonte: Autores.

Gráfico 1. Densidade dos filmes Carestream e AGFA.

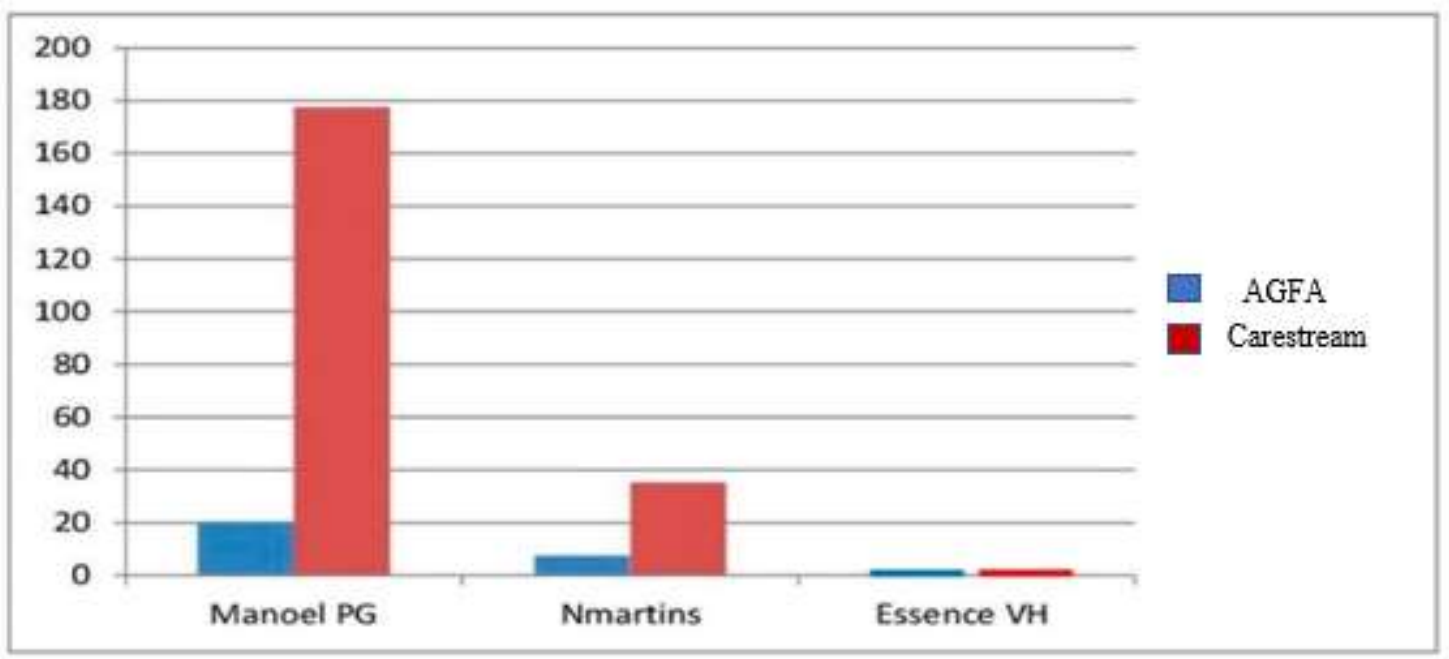

Fonte: Autores.

\section{Discussão}

Erros em radiografias dentárias são muito comuns e estão relacionados, principalmente, ao posicionamento do paciente, sobreposições anatômicas, assim como, alterações de densidade e processamento de imagens (Akarslan et al., 2003; Ghazali, Mohd, \& Norman, 2021; Eid \& Magalhães Filho, 2017). É fundamental o processamento químico do filme na formação da imagem radiográfica para complementar o diagnóstico (Gasparini et al., 2005; Casanova, 2002), visto que o objetivo da radiologia odontológica é produzir uma imagem que apresente detalhes suficientes (Santiago et al, 2011; Woitchunas et al., 2001). Esse processo pode ser realizado de forma manual, por meio da imersão do filme em soluções químicas, consistindo em cinco etapas: revelação, lavagem intermediária, fixação, lavagem final e secagem. (Lacerda \& Cordeiro, 2019)

As propriedades dos filmes radiográficos como sensibilidade, densidade, contraste, nitidez e latitude em uma imagem radiográfica são influenciadas pelas características de cada filme, fatores de exposição e condições de processamento. 
No processamento radiográfico, que deve ser realizado sob condições controladas e padronizadas, com atenção cuidadosa aos detalhes (Larentis et al, 2009), os tipos de soluções reveladoras, degradação, exaustão, método de processamento utilizado, visual e temperatura x tempo e lavagem final, influenciam na qualidade da imagem. Quando da comparação visual do filme Carestream e AGFA no controle e na câmara escura Delta sem visor, o comportamento desses em relação à DVB foi diferente. O filme AGFA mostrou-se com densidade e escurecimento maior, sendo estatisticamente significativo, de acordo com a Tabela 1.

Poucos estudos relacionam as câmaras escuras portáteis utilizadas de rotina nos consultórios odontológicos com a filtração de luz ambiente e a interferência nas imagens diagnósticas. Devido ao aumento da sensibilidade dos filmes radiográficos, buscando-se um menor tempo de exposição aos raios X (Araujo et al., 2018), os profissionais têm procurado utilizar os do grupo E ou E/F, mais sensíveis a radiação X e a luz, entretanto essa propriedade interfere no velamento. Nesse estudo, as câmaras escuras ManoelPG e NMartins, ambas com visor, apresentaram velamento à luz ambiente, constatado pelo contraste e densidade da imagem da moeda, sendo observado diferença significativa entre as câmaras e os filmes conforme na tabela 1 e figura 4. Na câmara escura EssenceVH ocorreu velamento total, não sendo possível visualizar a imagem da moeda, assim como mensurar a densidade, apresentando-se totalmente escura.

De acordo com Pardini, Machado e Watanabe (1999) a maioria dos profissionais, na sua rotina clínica, não prioriza o procedimento radiográfico. Deveriam se preocupar com a escolha do filme, do modelo da câmara escura, soluções processadoras de qualidade e padronização nas etapas do processamento radiográfico. No estudo em questão, foi verificado que as câmaras portáteis com visor não filtraram adequadamente a luz causando velamento radiográfico. Corroborando o estudo de Takeshita et al. (2011), que também verificou marcas comerciais de filmes radiográficos com diferentes câmaras escuras portáteis.

Apesar dos avanços nos sistemas digitais, as técnicas radiográficas convencionais e as câmaras escuras portáteis são amplamente utilizadas em consultórios odontológicos no Brasil. Esse fato ocorre devido ao baixo custo, praticidade e agilidade do processo (Miguel, et al., 2014). Entretanto, estudos relacionados a materiais para a confecção de câmaras devem ser realizados, no intuito de bloquear ou diminuir a passagem de luz para não prejudicar a qualidade da imagem e consequentemente o diagnóstico.

\section{Conclusão}

A câmara escura sem visor mostrou-se adequada para o processamento radiográfico, entretanto, as com visor ou totalmente acrílica não filtraram adequadamente a luz causando velamento.

\section{Referências}

Akarslan, Z. Z., Erten, H., Gungor, K., \& Celik, I. (2003). Common errors on panoramic radiographs taken in a dental school. Journal of Contemporary Dental Practice, 4: 19-26.

Araújo, D. D., Araújo, D. D., Gazolla, M. S., Dominguete, M. H. L., Oliveira, L. A. M. de, Graciano, K. P. P., \& Oliveira, C. M. G. de. (2018). Conhecimento do cirurgião dentista sobre filmes radiográficos interferindo na otimização do processamento radiográfico. Archives of health investigation, 7(8).

Brasil. Resolução - RDC No 330, de 20 de dezembro de 2019. Diário oficial da união.

Casanova, M. L. S. (2002). Análise comparativa das variações de tempos de exposição, tipo de processamento e do efeito da degradação das soluções processadoras na qualidade da imagem radiográfica. Tese de doutorado, Universidade Estadual de Campinas, São Paulo, Piracicaba, SP, Brasil.

Eid, N. L. M., \& Magalhães F. G. (2017). Erros na Aquisição de Radiografias Periapicais Realizadas por Acadêmicos do Centro Universitário UNIRG. Revista da $A c B O, 26(1): 14-20$.

Filho, A. F. S., Lucas, J. M. S., Silva, E. N. D., Nogueira, D. N., Cruz, M. R. S., \& Neta, N. B. D. (2021). Análise de erros radiográficos em clínica de odontopediatria de uma instituição de ensino superior de Teresina - Piauí. Arquivos Em Odontologia, 57, 46-56. 
Research, Society and Development, v. 10, n. 9, e49910918467, 2021 (CC BY 4.0) | ISSN 2525-3409 | DOI: http://dx.doi.org/10.33448/rsd-v10i9.18467

Gasparini, A. L., Lemke, F., Carvalho, A. S., Cunha, F. L., Junqueira, J. L. C, \& Tavano, O. (2005) Verificação das condições de processamento radiográfico em consultórios odontológicos. RGO: Revista Gaúcha Odontol. 53(3):217-9

Ghazali, L., Mohd, Y. M. Y. P., \& Norman, N. H. (2020) Effects of scanning parameters reduction in dentalradiographs on image quality and diagnostic performance: A randomised controlled trial. Journal Orthodontic, Mar;48(1):5-12.

Januário, B. C., Carvalho, L. F., \& Larentis, N. L. (2020). Avaliação do nível de conhecimento sobre processamento radiográfico dos acadêmicos nas fases finais do curso de odontologia da Ulbra-Torres. Stomatos, 26(51);110-6.

Lacerda, L. J. R., \& Dirceu, A. C. D. A. J. (2019). Recipiente proveniente de material reutilizado como alternativa sustentável para processamento radiográfico manual. Revista da Universidade Vale do Rio Verde. $1 ; 1$.

Larentis, N. L., Carlos, M. E. W., Barbosa, A. N., Fontanella, V. R. C., \& Matos, A. P. (2009). A prática do processamento radiográfico em odontologia: uma análise qualitativa. Revista da ABENO, 9(1):39-43.

Lemke, F., Lopes, D. A. Z., Tavano, O., \& Mezadri, A. C. (2006). Análise das Condições de Exposição e Processamento Radiográficos em Consultórios Odontológicos Quanto ao Velamento. Revista da Faculdade de Odontologia de Porto Alegre, 47(1):5-8.

Lima, F. O., Gominho, L. F., Penha, E. S., Alameida, M. S. C., Teodoro, M. K. R., Santos, T. A., et al (2021). Principais erros encontrados em radiografias descartadas na clínica-escola de uma instituição de ensino superior. Research, Society and Development, $10(1) ;$ e23110111689.

Miguel, C., Barros, F. S., Lendzion, R. S., \& Rocha, A. S. P. S. (2014, outubro). Avaliação da densidade ótica de base mais véu de filmes intraorais processados em caixas portáteis de revelação. Congresso Brasileiro de Engenharia Biomédica, Uberlândia, Minas Gerais, Brasil, XXIV.

Moreira, H. L. O., Marcylene, A. B. M. A., \& Larentis, N. L. (2017). Preferências dos docentes de um curso de Odontologia quanto à forma de visualização de radiografias digitais. Revista da ABENO. 17(1).

Pardini, L. C., Machado, L. G., \& Watanabe, P. C. A. (1999). Avaliação da qualidade de radiografias obtidas na FOR/USP - Processamento Radiográfico. I Congresso Interno de Pesquisa Faculdade de Odontologia de Ribeirão Preto, USP, 1;138.

Santiago, M. C., Guimarães, C. S., Silveira, M. M. F., Pontual, M. L. A., Estrela, C., \& Rodrigues, C. D (2011). Avaliação do filtro de luz das câmaras escuras portáteis e de sua influência na qualidade da imagem radiográfica. Dental Press Endodontic. 3:45-50.

Silva, J. M. F., Oliveira, L. C., Daroz, B. G., Peyneau, P. D., Pereira, T. C. R., \& Vaz, S. L. A. (2016). Erros cometidos pelos estudantes de radiologia de uma universidade pública brasileira na realização de radiografias periapicais. Revista da ABENO. 16(1):99-109.

Silva, M. C., Silva, M. C., Silva, I. S. N., \& Mania, T. V. (2017). Análise da qualidade da imagem e arquivamento de radiografias periapicais. Arquivos Em Odontologia, 53: e18.

Tamburús, J. R., Lavrador, M. A. S, \& Oliveira, A. C. (1999). Radiographic processing - portable darkroom: relation between light filtering and base density plus fog. Revista Odontologia da Univesrsidade de São Paulo, 13(1);93-100.

Takeshita, W. M., Iwaki, L. C. V., Silva, M. C., Pieralisi, N., Yamashita, A. L., \& Zeczkowski, M. (2011). Avaliação digital da densidade base velamento e contraste obtidos de filmes radiográficos periapicais processados em diferentes câmaras escuras portáteis. Revista Brasileira de Odontologia, 68(2):200-4.

Watanabe, P. C. A., \& Arita, E. S. Imaginologia e Radiologia Odontológica. Elsevier Editora, 2013

Woitchunas, G. F. P., Veeck, E. B., Holderbaum, R. M., \& Souza, P. H. C. (2001). Avaliação in vitro da sensibilidade de três filmes radiográficos. Revista da Faculdade de Odontologia de Passo Fundo, 6(2);61-65. 